\title{
ÓLEO DA POLPA DE MACAÚBA COMO COLETOR ALTERNATIVO NA FLOTAÇÃO SELETIVA ENTRE APATITA E CALCITA DE ORIGEM SEDIMENTAR
}

\author{
Rayander Martins Pimenta ${ }^{1 *}$ \\ Jéssica Vicente Luiz' \\ Everton Pedroza dos Santos ${ }^{2}$
}

\section{Resumo}

O beneficiamento de minérios fosfáticos por flotação é o processo mais eficiente utilizado para separar a apatita dos minerais da ganga, como calcita, dolomita e quartzo. Entretanto, a similaridade das propriedades superficiais da apatita e calcita geralmente leva à adsorção não seletiva de reagentes, tornando a separação seletiva desses minerais um problema mundial. O fosfato sedimentar, estudado no presente trabalho, apresenta uma composição mineralógica complexa, contendo urânio associado ao mineral da apatita e como principais minerais de ganga, calcita e quartzo. Este trabalho tem como objetivo investigar a potencial aplicação do óleo de polpa de macaúba (Acrocomia Aculeata) como coletor alternativo na separação seletiva entre apatita e calcita de origem sedimentar, por meio de experimentos de microflotação em tubo de Hallimond. Os resultados mais relevantes foram obtidos utilizando uma concentração de óleo de polpa de macaúba de $200 \mathrm{mg} . \mathrm{L}^{-1} \mathrm{em}$ $\mathrm{pH}$ 10,5, onde a apatita apresentou flotabilidade superior a 95\%. A calcita, no entanto, apresentou baixa flotabilidade em todos os experimentos realizados, com e sem o uso de amido de milho como depressor, sugerindo uma concordância com os resultados da literatura, onde o amido de milho é eficiente apenas para a depressão da calcita de origem ígnea.

Palavras-chave: Apatita; Calcita; Flotação seletiva; Macaúba.

\section{MACAÚBA PULP OIL AS AN ALTERNATIVE COLECTOR IN THE SELECTIVE FLOTATION OF APATITE AND CALCITE FROM SEDIMENTARY ORIGIN}

\begin{abstract}
The beneficiation of phosphate ores by froth flotation is the most efficient process used to separate apatite from gangue minerals like calcite, dolomite and quartz. However, the similarity of surface properties between apatite and calcite generally lead to non-selective adsorption of reagents, rendering the selective separation of these minerals a worldwide problem. The sedimentary phosphate, studied in the present work, presents a complex mineralogical composition having uranium associated with the apatite mineral and as the main gangue minerals calcite and quartz. This work aims at investigating the potential application of macauba (Acrocomia Aculeata) pulp oil as an alternative colector in selective separation of apatite and calcite from sedimentary origin, through microflotation experiments conducted in Hallimond tube. The most interesting results were

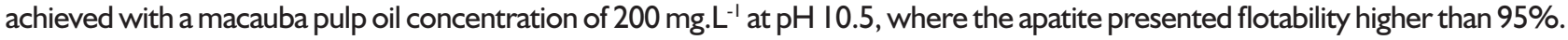
Calcite, however, showed low flotability in all experiments, with and without the use of corn starch as depressant, suggesting an agreement with the results of the literature, where corn starch was efficient only for depression of calcite from igneous origin. Keywords: Apatite; Calcite; Selective flotation; Macaúba.
\end{abstract}

\section{INTRODUÇÃO}

A apatita $\left[\mathrm{Ca}_{5}(\mathrm{PO} 4)_{3}(\mathrm{~F}, \mathrm{OH})\right]$ e a calcita $\left[\mathrm{CaCO}_{3}\right]$ são minerais presentes em minérios fosfáticos encontrados em diferentes ambientes geológicos, sendo de grande aplicação na fabricação de produtos comerciais [I]. Esses minerais são hidrofílicos e apresentam características físicoquímicas bastante similares devido a composição química, sendo um desafio a obtenção de uma boa separabilidade entre eles [2].

'Engenharia Metalúrgica, Centro Federal de Educação Tecnológica Celso Suckow da Fonseca - CEFET/RJ, Angra dos Reis, RJ, Brasil.

2Engenharia Metalúrgica e de Materiais, Departamento de Engenharia Metalúrgica, Centro Federal de Educação Tecnológica Celso Suckow da

Fonseca - CEFET/RJ, Angra dos Reis, RJ, Brasil.

*Autor correspondente: rayander.pimenta@aluno.cefet-rj.br

2176-1523 (C) 2020. Pimenta et al. Publicado pela ABM. Este é um artigo publicado em acesso aberto (Open Access) sob a licença Creative Commons Attribution, que permite uso, distribuição e reprodução em qualquer meio, sem restrições desde que o trabalho original seja corretamente citado. 
O processo de flotação é um dos principais métodos de separação utilizado para a concentração deste tipo de minério [3]. A flotação direta da apatita é normalmente realizada na faixa alcalina de $\mathrm{pH}$, sendo empregados coletores aniônicos e depressores para a calcita [4]. Assim, os coletores aniônicos mais utilizados são os ácidos graxos de cadeia longa e seus sais alcalinos, principalmente oriundos de óleos vegetais ricos em ácido oleico [5]. Como depressores, são utilizados taninos, quebrachos, policrilamidas, silicato de sódio, dextrinas, e principalmente $o$ amido, pois apresenta baixo custo e fornece uma melhor eficiência na depressão da calcita [6].

Estudos de flotabilidade da apatita e calcita das minas de Cajati-SP (origem ígnea) e Itataia-CE (origem sedimentar), utilizando o oleato de sódio como coletor da apatita e o amido de milho como depressor da calcita, na faixa alcalina de $\mathrm{pH}$, mostraram a obtenção de boa seletividade para os minerais da mina de Cajati-SP, onde a apatita foi flotada e a calcita deprimida, enquanto que para os minerais oriundos da mina de Itataia-CE, a rota de processo não se mostrou eficiente [7].

Diversos estudos vêm sendo realizados visando a separação seletiva entre minerais presentes em minérios fosfáticos de origem sedimentar, utilizando óleos vegetais como coletores aniônicos [8-10]. O óleo de macaúba, tanto da polpa quanto da castanha, também tem sido empregado com sucesso como coletor alternativo nos minérios fosfáticos brasileiros [ I I- I4]. O óleo extraído da polpa de macaúba tem um grande potencial de utilização como coletor alternativo da apatita, pois é rico em ácidos graxos insaturados, conforme pode ser visto na Tabela I [I5].

Desta forma, este trabalho tem como objetivo avaliar a eficiência do óleo da polpa de macaúba como reagente alternativo para a flotação direta da apatita de origem sedimentar, na presença e na ausência do amido de milho como depressor.

\section{MATERIAIS E MÉTODOS}

No presente trabalho foram utilizadas amostras de apatita e calcita de origem sedimentar do nordeste brasileiro. As amostras foram cominuídas em grau de porcelana e depois peneiradas para obtenção de alíquotas na faixa granulométrica entre $-140+200 \#(-105+74 \mu \mathrm{m})$. Nos experimentos, as amostras de apatita e calcita foram flotadas separadamente em um tubo de Hallimond modificado utilizando os parâmetros apresentados na Tabela 2, que são semelhantes aos parâmetros utilizados por Silva et al. [I I]. As principais diferenças entre os procedimentos estão no tempo de condicionamento e na faixa granulométrica dos minerais.

O procedimento de saponificação do óleo da polpa de macaúba foi semelhante ao realizado por Santos et al. [10]. Porém, a saponificação foi realizada à quente em $80^{\circ} \mathrm{C}$ durante 20 minutos, utilizando uma relação de $\mathrm{NaOH}$ /óleo de 6:I, não sendo alcoólica. Como reagente depressor da calcita, foi utilizado o amido de milho da marca Calbiochem, gelatinizado à quente em $84^{\circ} \mathrm{C}$ durante 5 minutos, utilizando uma relação de amido/ $\mathrm{NaOH}$ de $4: 1$. Todos os reagentes (coletores e depressor) utilizados no presente estudo foram preparados com concentração $1000 \mathrm{mg} \cdot \mathrm{L}^{-1}$ para posteriormente serem diluídas nas concentrações desejadas. Para a regulagem do $\mathrm{pH}$ no estudo foram usados o ácido clorídrico e o hidróxido de sódio da marca Alphatec, ambos em concentração de $0, I M$.

Os resultados obtidos na flotabilidade da apatita e da calcita utilizando o óleo da polpa de macaúba foram comparados com oleato de sódio da marca VETEC, que foi saponificado utilizando a mesma metodologia do óleo de polpa da macaúba.

\section{RESULTADOS E DISCUSSÃO}

Os ensaios de flotabilidade da apatita e calcita foram realizados visando uma flotação direta, de modo a se obter uma elevada separabilidade entre os minerais. As Figuras Ia e Ib apresentam a flotabilidade da apatita e da calcita em função da concentração do óleo da polpa de macaúba saponificado e do oleato de sódio, respectivamente, em pH 10,5.

Com base na Figura la, é possível observar que a flotabilidade da apatita apresentou um aumento considerável entre as concentrações de 10 e $200 \mathrm{mg} \cdot \mathrm{L}^{-1}$, passando de 41 , I \% para $96,6 \%$, enquanto que nas mesmas condições a flotabilidade da calcita se manteve praticamente inalterada, variando entre $80,8 \%$ e $83,4 \%$. Já na Figura Ib, a apatita apresentou um aumento na flotabilidade de $44,0 \%$ para $84,6 \%$ entre as concentrações de 10 e $200 \mathrm{mg}^{\mathrm{L}^{-1}}$, respectivamente.

Tabela I. Porcentagem de ácidos graxos do óleo da polpa de Macaúba

\begin{tabular}{lc}
\hline \multicolumn{1}{c}{ Ácido Graxo } & Porcentagem (\%) \\
\hline Palmítico $(\mathrm{Cl} 6: 0)$ & $14,87 \pm 0,60$ \\
Palmitoleico $(\mathrm{Cl} 6: \mathrm{I})$ & $3,96 \pm 0,39$ \\
Esteárico $(\mathrm{Cl} 8: 0)$ & $1,09 \pm 0,004$ \\
Oleico $(\mathrm{Cl} 8: \mathrm{I})$ & $67,36 \pm 1,12$ \\
Linoleico $(\mathrm{Cl} 8: 2)$ & $12,16 \pm 0,02$ \\
Linolênico $(\mathrm{Cl} 8: 3)$ & $0,54 \pm 0,08$ \\
\hline
\end{tabular}

Fonte: Adaptado de Colonelli e Silva [15].

Tabela 2. Parâmetros operacionais utilizados em um tubo de Hallimond

\begin{tabular}{lc}
\hline \multicolumn{1}{c}{ Condições } & Valores \\
\hline Vazão de ar $\left(\mathrm{mL}\right.$ min $\left.^{-1}\right)$ & 40 \\
Tempo de flotação $(\mathrm{min})$ & $\mathrm{I}$ \\
$\begin{array}{l}\text { Tempo de condicionamento do } \\
\text { depressor (min) }\end{array}$ & 5 \\
$\begin{array}{l}\text { Tempo de condicionamento do } \\
\text { coletor (min) }\end{array}$ & 5 \\
Massa do mineral $(\mathrm{g})$ & $\mathrm{I}$ \\
Faixa granulométrica dos minerais $(\#)$ & $-140+200 \#(-105+74 \mu \mathrm{m})$ \\
\hline
\end{tabular}



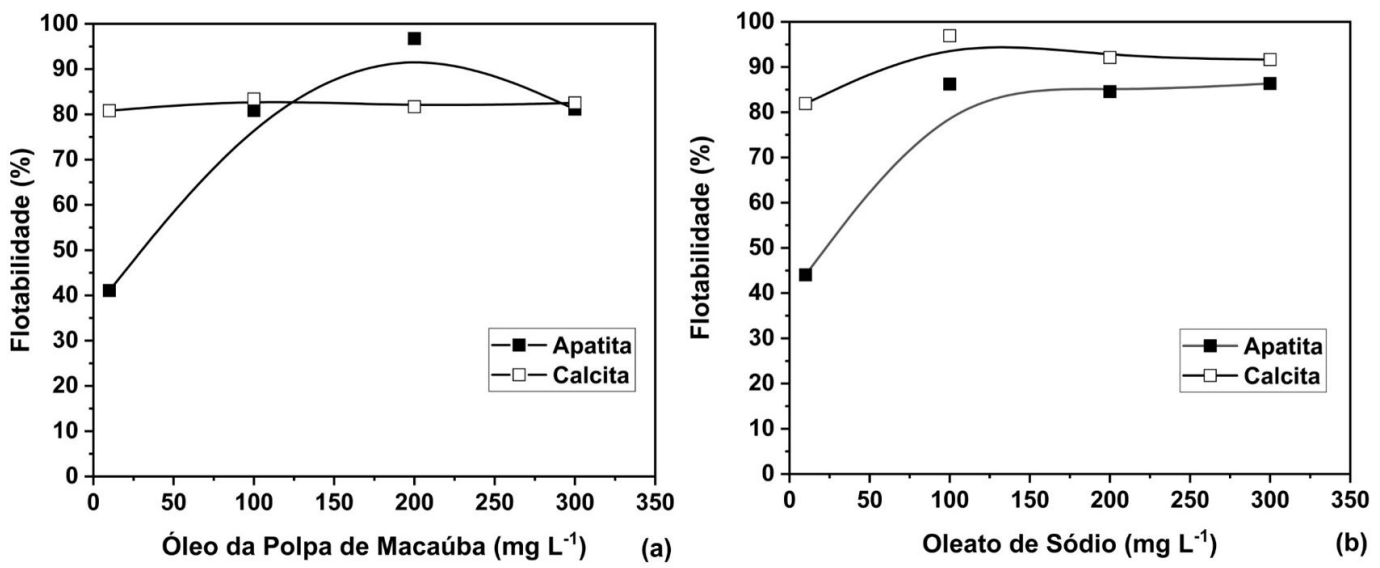

Figura I. Flotabilidade da apatita e da calcita em pH 10,5, variando a concentração do óleo da polpa de macaúba (a) e a concentração do oleato de sódio (b) como coletores.

A calcita obteve um aumento na flotabilidade, variando de $82,0 \%$ na concentração de $10 \mathrm{mg} \cdot \mathrm{L}^{-1}$ para $92,0 \%$ na concentração de $300 \mathrm{mg} \cdot \mathrm{L}^{-1}$.

Comparando-se os resultados das Figuras la e Ib, é possível observar que a flotabilidade da apatita apresentou comportamento similar na presença de ambos os coletores (óleo da polpa de macaúba e oleato de sódio), alcançando valores elevados de flotabilidade em concentrações elevadas dos coletores. Enquanto a flotabilidade da calcita, na presença do oleato de sódio, foi superior aos resultados obtidos quando utilizado o óleo da polpa de macaúba como coletor. As diferenças observadas nas Figuras la e Ib possivelmente se devem ao fato de o óleo da polpa de macaúba ser composto, além do ácido oleico, por outros ácidos graxos de cadeia longa, como visto na Tabela I.

Estudos realizados por Pachêco et al. [13] apresentaram resultados semelhantes de flotabilidade da apatita na presença do óleo da polpa de macaúba, na faixa alcalina de $\mathrm{pH}$. Entretanto, no estudo em questão [13], os autores mostraram ser possível obter elevada recuperação da apatita na presença de uma baixa dosagem $\left(2,5 \mathrm{mg} \cdot \mathrm{L}^{-1}\right)$ do óleo de polpa de macaúba. Essa diferença na dosagem do óleo da polpa de macaúba ocorreu possivelmente devido a diferença do tipo de apatita estudado, que deve ser de origem ígnea, enquanto a apatita utilizada neste estudo, é de origem sedimentar.

Com intuito de aumentar a seletividade entre a apatita e a calcita, foram realizados estudos utilizando amido de milho como depressor da calcita. A Figura 2 apresenta os resultados de flotação da apatita e da calcita em função da concentração do amido de milho, utilizando-se $256,5 \mathrm{mg} \cdot \mathrm{L}^{-1}$ do óleo da polpa de macaúba como coletor, em pH 10,5. É possível observar que ocorre um decréscimo da flotabilidade da calcita à medida que aumenta a concentração do amido, tendo assim um valor mínimo de flotabilidade de $57,8 \%$ na concentração de $150 \mathrm{mg} \cdot \mathrm{L}^{-1}$.

Com base na literatura [16], o efeito depressor do amido sobre a superfície da calcita pode estar relacionado

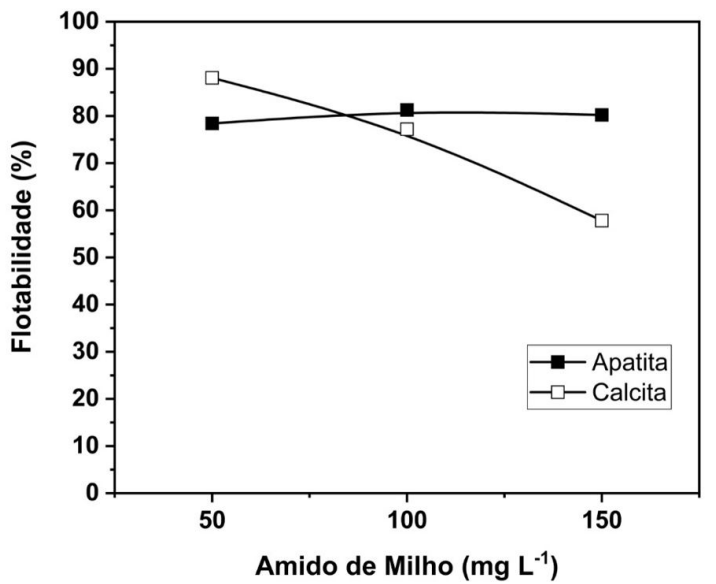

Figura 2. Flotabilidade da apatita e da calcita em função da concentração do amido como depressor em $\mathrm{pH}$ 10,5.

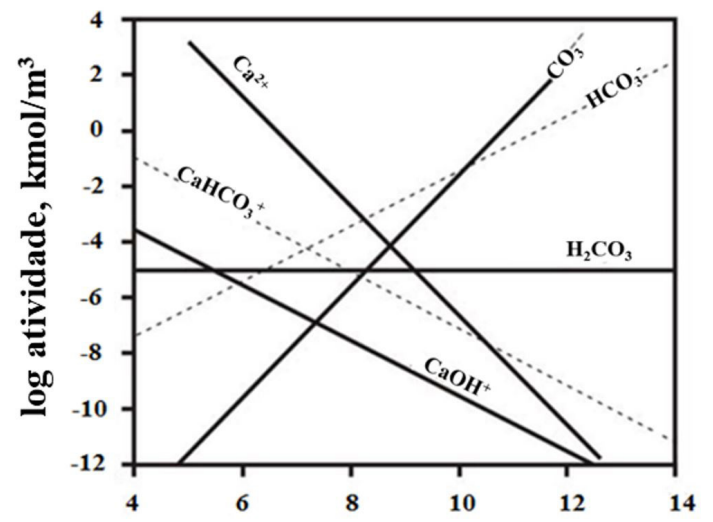

Figura 3. Diagrama de distribuição das espécies da calcita. Adaptado de Hanna e Somasundaran [16].

à formação de compostos químicos, entre ele e os íons cálcio. Analisando-se a Figura 3, é possível observar que em pH 10,5 a concentração dos íons $\mathrm{Ca}^{2+}$ e $\mathrm{CaOH}^{+}$é baixa, quando comparada com a faixa de $\mathrm{pH}$ neutra. Deste modo, a redução da flotabilidade da calcita em pH 10,5, 

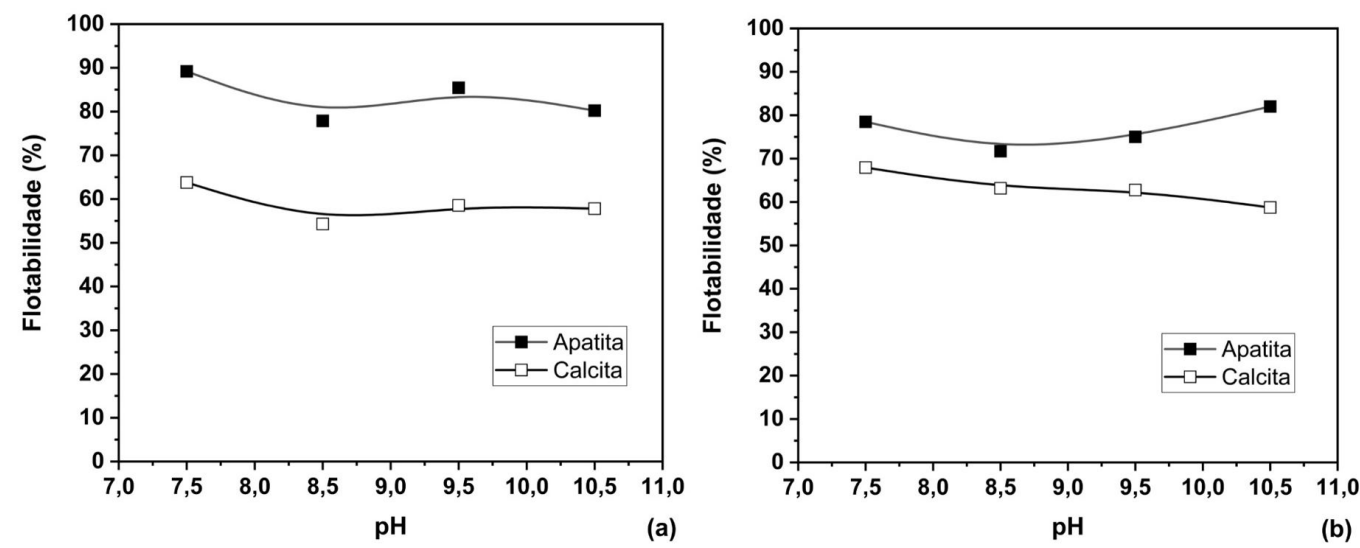

Figura 4. Flotabilidade da apatita e da calcita em função da variação do $\mathrm{pH}$ utilizando-se $256,5 \mathrm{mg} \cdot \mathrm{L}^{-1}$ do óleo da polpa de macaúba como coletor e $150 \mathrm{mg} . \mathrm{L}^{-1}$ do amido como depressor (a) e utilizando-se $256,5 \mathrm{mg} . \mathrm{LL}^{-1}$ do oleato de sódio como coletor e I50 mg. $\mathrm{LL}^{-1}$ do amido como depressor (b).

observada na Figura 2, pode estar relacionada à diminuição da quantidade de íons cálcio necessários para a adsorção do amido na superfície da calcita.

Com o intuito de melhorar o efeito depressor do amido sobre a superfície da calcita, foram realizados ensaios de flotabilidade variando o $\mathrm{pH}$, na presença de $150 \mathrm{mg} \cdot \mathrm{L}^{-1}$ do amido de milho, utilizando-se $256,5 \mathrm{mg} . \mathrm{L}^{-1}$ do óleo da polpa de macaúba e do oleato de sódio como coletores, apresentados nas Figuras 4a e 4b, respectivamente.

$\mathrm{Na}$ Figura 4a é possível observar que a porcentagem de flotado da apatita e calcita se manteve quase inalterada, onde a apatita permaneceu entre as faixas de $80,0 \%$ e $90,0 \%$ e a calcita entre $54,0 \%$ e $64,0 \%$. Já na Figura $4 \mathrm{~b}$ em pH inferior a 8,5 a apatita mostrou uma estabilização de sua flotabilidade e a calcita apresentou uma pequena variação com a elevação do $\mathrm{pH}$, passando de 59,0\% para 70\% entre os pH's 10,5 e 7,5, respectivamente. $\mathrm{Na}$ faixa neutra de $\mathrm{pH}$, em ambos os sistemas de reagentes, na presença do amido como depressor, não ocorreu a diminuição da flotabilidade da calcita, possivelmente, relacionado à baixa adsorção do amido em sua superfície.

Com base nas Figuras 4a e 4b, observa-se que na faixa de $\mathrm{pH}$ neutra, os resultados não foram condizentes com a literatura [16], que indicava a depressão da calcita devido à presença de uma maior concentração de íons $\mathrm{Ca}^{2+}$ e $\mathrm{CaOH}^{+}$. Porém, estudos realizados por Assis et al. [7], da flotabilidade da apatita e calcita das minas de Cajati-SP (origem ígnea) e ltataia-CE (origem sedimentar), mostraram que a separação entre os minerais de origem sedimentar não foi eficiente, visto que a calcita apresentou elevada flotabilidade devido à baixa adsorção do amido neste mineral. Desta forma, os resultados do presente estudo confirmam os resultados obtidos por Assis et al. [7], visto que o amido de milho não se mostrou um bom depressor para a calcita de origem sedimentar. Os autores [7] atribuíram este comportamento às diferenças nas características químicas, físicas e mineralógicas dos minerais de origem sedimentar, tais como a rugosidade da superfície, tamanho de grão e contaminação das superfícies minerais por átomos de ferro.

\section{CONCLUSÃO}

Os estudos de microflotação realizados em tubo de Hallimond modificado usando o óleo da polpa de macaúba saponificado como coletor da apatita apresentaram resultados satisfatórios, visto que a flotação deste mineral foi superior a 95,0\% na concentração de $200 \mathrm{mg} \cdot \mathrm{L}^{-1}$ na faixa de $\mathrm{pH}$ alcalino. A maior dosagem do óleo da polpa de macaúba, em comparação com a literatura, para obter uma elevada flotabilidade da apatita, se deve possivelmente à origem de apatita (sedimentar) utilizado no presente estudo.

O amido de milho não se mostrou um bom depressor para calcita de origem sedimentar, visto que apresentou elevada flotabilidade, corroborando com os dados da literatura, o que sugere uma baixa adsorção do amido sobre a calcita. Então, para trabalhos futuros, objetivando uma melhor depressão para a calcita de origem sedimentar, sugere-se a utilização de outros reagentes depressores, tais como, taninos, quebrachos, policrilamidas, silicato de sódio e dextrinas.

\section{Agradecimentos}

Os autores agradecem ao CEFET/RJ Campus Angra dos Reis pelo apoio e infraestrutura, essenciais para $\circ$ desenvolvimento deste trabalho.

\section{REFERÊNCIAS}

I Loureiro, F.L, Monte, M. B.M., Nascimento, M. Agrominiras: fosfato em rochas \& minerais industriais: usos e especificações. 2. ed. Rio de Janeiro: CETEM; 2008. p. I4I - I74. 
Óleo da polpa de macaúba como coletor alternativo na flotação seletiva entre apatita e calcita de origem sedimentar

2 Sis $\mathrm{H}$, Chander S. Adsorption and contact angle of single and binary mixtures of surfactants on apatite. Minerals Engineering. 2003; 16(9):839-848.

3 Chaves AP. Máquinas de flotação. In: Chaves AP, editor. Flotação: o estado da arte no Brasil. Vol. 4. São Paulo: Signus; 2006.

4 Tanaka Y, Katayama N, Arai S. Reagents in phosphate flotation. In: Somasundaran P, Moudgil BM, editores. Reagents in mineral technology. Vol. 2. New York: Marcel Dekke; 1987. cap. 20, p. 645-662.

5 Oliveira MS, Peres AEC. Flotabilidade da apatita e minerais de ganga provenientes de minério sílico-carbonatado com oleato de sódio. Revista Escola de Minas. 2010;63(3):55I-557.

6 Guimarães RC, Araújo AC, Peres AEC. Reagents in igneous phosphate ores flotation. Minerals Engineering. 2005; 18(2): 199-204.

7 Assis SM, Viana SHM, Silva JM. Calcitas, dolomitas e apatitas: algumas características inerentes $\times$ microflotação. In: Anais do II Encontro do Hemisfério Sul sobre Tecnologia Mineral; XII Encontro Nacional de Tratamento de Minérios e Hidrometalurgia; 1987; Rio de Janeiro. Vol. I. Rio de Janeiro: Editora ABM; 1987. p. 265-280.

8 Costa DS. Uso de óleos vegetais amazônicos na flotação de minérios fosfáticos [tese]. Belo Horizonte: PPGEM/ UFMG; 2012.

9 Costa DS, Oliveira JE Jr. Óleo residual de fritura como reagentes coletores na flotação de minério fosfático. São Paulo: UNIFESSPA; 2015.

10 Santos EP, Dutra AJB, Oliveira JF. The effect of jojoba oil on the surface properties of calcite and apatite aiming at their selective flotation. Minerals Engineering. 20I5; I34:34-38.

II Silva AC, Silva EMS, Silva TFV, Ferreira KC. Influência do método de saponificação na microflotação de apatita utilizando óleo da castanha de macaúba. Tecnologica em Metalurgia, Materiais e Mineração. 2017;14(I):30-38.

12 Pachêco CAT. Análise técnica e econômica do uso do óleo da polpa de macaúba (Acrocomia aculeata) como coletor na flotação [dissertação]. Goiânia: UFG; 2016.

I3 Pachêco CAT, Silva AC, Silva EMS, Rocha TWP. Microflotação de apatita com óleos da polpa e da castanha de macaúba. In: Neves AF, Paula MH, Anjos PHR, Silva AG, editores. Estudos Interdisciplinares em Ciências Biológicas, Saúde, Engenharias e Gestão. I. ed. Vol. I. São Paulo: Blucher; 2016. p. 249-260.

I4 Silva AC, Silva EMS, Rocha TWP. Microflotação de apatita utilizando óleo da castanha de macaúba (Acrocomia aculeata) como coletor. Tecnologica em Metalurgia, Materiais e Mineração. 20 I5; I2(2): I 46- 152.

15 Colonelli TA, Silva C. Estratificação heterogênea dos ácidos graxos livres do óleo da polpa de macaúba (Acrocomiaaculeata) em etanol. Revista Semina: Ciências Exatas e Tecnológica. 20l4;35(I):25-38.

16 Hanna HS, Somasundaran P. Flotation of Salt-type minerals. In: Fuerstenau MC, editor. Flotation: A. M. Gaudin memorial volume. New York: AIME; 1976. p. 197-272.

Recebido em: 15 Fev. 2019

Aceito em: 22 Jul. 2020 